\title{
The Influence of Multiple Fluorination on the Ring Opening of [2.2.2]Propellane
}

\author{
Ivana Antol, Mirjana Eckert-Maksić
}

\author{
Division of Organic Chemistry and Biochemistry, Ruđer Bošković Institute, P.O.B. 180, Zagreb HR-10002, Croatia \\ Authors' e-mail addresses: iantol@irb.hr, mmaksic@irb.hr
}

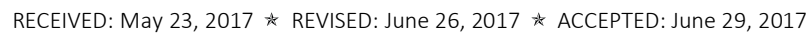

THIS PAPER IS DEDICATED TO PROF. MIRJANA METIKOŠ-HUKOVIĆ ON THE OCCASION OF HER BIRTHDAY

\begin{abstract}
Effect of electronegative substituents on ring opening of [2.2.2]propellane was examined by quantum chemical calculations at CASSCF and CASPT2 level of theory. Comparison of the results with those obtained with MR-AQCC calculations indicated that calculations beyond CASSCF level were needed for reliable description of the process. Calculations show a strong effect of fluorination on the central bond elongation energy profile, with the effect being most pronounced for the perfluoro derivative. Moreover, for rearrangement of the most of multiply fluorinated derivatives, including perfluoro[2.2.2]propellane, CASPT2 calculations predict a single minimum potential energy well, which hampers rearrangement to significantly more stable fluorinated 1,4-dimethylenecyclohexane analogues. This observation is in harmony with high stability of synthetically available stable heavily fluorinated derivatives which, in contrast to the parent [2.2.2] propellane, do not rearrange to the thermodynamically most stable fluorinated 1,4-dimethylene-cyclohexane isomer.
\end{abstract}

Keywords: Ab initio calculations, Grob rearrangement, isomerisation, [2.2.2]propellane, fluorination.

\section{INTRODUCTION}

T HE publication of W. D. Stohrer's and R. Hoffmann's 1972 seminal paper on bond-stretch isomerism of [2.2.2]propellane inspired a considerable interest in this system. ${ }^{[1]}$ Since then, this topic was addressed by many theoretical studies varying in the computational level from INDO ${ }^{[2]}$ and SCF calculations, ${ }^{[3,4]}$ DFT tests ${ }^{[5-7]}$ to more demanding CASSCF and MP2-CASSCF. ${ }^{[8,5]}$ Our own computational studies in this area using MR-AQCC method[9] showed that the conventional closed isomer $1 \mathrm{a}$ and its 3.6 $\mathrm{kcal} \mathrm{mol}^{-1}$ more stable open diradical form $\mathbf{1 b}$ were separated by barrier as high as $22.7 \mathrm{kcal} \mathrm{mol}^{-1}$ and that the stability of $\mathbf{1 b}$ is artificial since it undergoes barierless cageopening (Grob fragmentation ${ }^{[10]}$ ) to the thermodynamically more stable 1,4-dimethylenecyclohexane 1c. Concomitantly, it was concluded that the bond-stretch isomerism does not occur in this system and emphasized that the [2.2.2]propellane might be a potentially stable compound due to the energy barrier for $\mathrm{C} 1-\mathrm{C} 4$ bond stretching which protects it from the Grob fragmentation process.

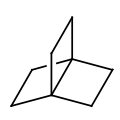

$1 \mathrm{a}$<smiles>C1CC2CCC1C2</smiles>

$1 b$<smiles>C=C1CCC(=C)CC1</smiles>

$1 \mathrm{c}$
In contrast to conclusion reached by theoretical considerations, 1a has not been directly observed, although generation of 1a by photocyclisation of $1 \mathrm{c}$ has been claimed by Dannenberg et al. ${ }^{[11]}$ The studies suggesting formation of [2.2.2]propellane by treatment of bridgehead dihalides with reducing agents were reinterpreted in a way that $1 \mathbf{a}$ is not mandatory intermediate. ${ }^{[12]}$ The first isolated and characterized [2.2.2] propellane derivative $\mathbf{2 a}^{[13]}$ was prepared by ultraviolet irradiation of the corresponding diazo ketone. In accordance to the mechanism based on the calculations, it undergoes Grob-type fragmentation to a mixture of amido 1,4-dimethylenecyclohexane derivatives $2 \mathrm{c}$ and 2d. Activation energy for this process was estimated to $\sim 22$ $\mathrm{kcal} \mathrm{mol} \mathrm{m}^{-1}$ and measured half-time of $2 \mathrm{a}$ was about $28 \mathrm{~min}$ at room temperature. It is interesting to note that recent 
attempt to prepare $\mathbf{2 a}$, as well as similar carboxyamido[2.2.2] propellane derivatives $3 \mathbf{a}$ and $4 \mathbf{a}$, by electroreduction of 1,4-dichlorobicyclo[2.2.2]octane-2-carboxamides failed.[14]

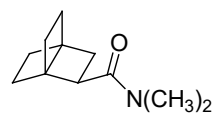

$2 a$<smiles>C=C1CCC(=CN(C)C)CC1</smiles><smiles>C=C1CCC(=C)C(N(C)C)C1</smiles>

2c 2d<smiles>O=C(C1CC2CCC1C2)N1CCCCC1</smiles>

3a<smiles>CN(C)C(=O)C1CC2(C(=O)N(C)C)CCC1CC2</smiles>

$4 a$
Further stabilisation of the [2.2.2]propellane moiety was achieved by fluorine substitution, as exemplified by synthesis of multiply fluorinated [2.2.2]propellanes $\mathbf{5 a}$ and $6 a$ by $[2+2]$ cycloadition to perfluorinated strained bicyclo[2.2.0]hex-1(4)-ene. ${ }^{[15,16]}$ The first among them (5a) rearranges to a mixture of the corresponding 1,4-dimethylenecyclohexanes with a half-time of 40 hours at $21^{\circ} \mathrm{C}$, while 6 a showed no signs of decomposition even after heating at $100{ }^{\circ} \mathrm{C}$ for more than 10 hours.

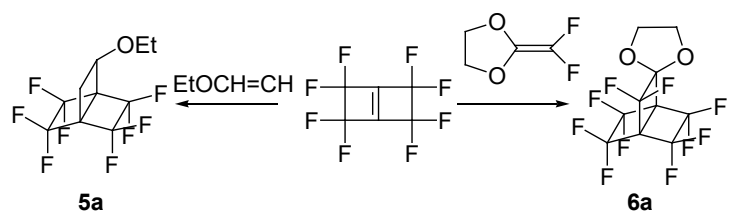

Clearly, substitution of [2.2.2]propellane framework with strong electron withdrawing substituents (fluorines and oxygens) leading to $\mathbf{5 a}$ and $\mathbf{6 a}$ strongly supresses Grobtype fragmentation. The remarkable stability of multiply fluorinated [2.2.2] propellane derivatives toward ring opening was mainly attributed to ability of fluorine atoms to stabilize four-membered ring system in the parent molecule ${ }^{[17]}$ thus increasing stability of the closed structure over open diradical isomer. Much less attention has been paid to the effect of fluorine atoms on stablitiy of the intermediate diradical formed upon central bond cleavage. In order to contribute to deeper understanding of the impact of fluorination on this process we have extended our previous computational mechanistic study on the parent [2.2.2]propellane isomerisation discussed in ref. [9] to molecules $6 a$, fluorinated $7 a-9 a$, perfluorinated $10 a$ and model systems $(\mathbf{1 1} \mathbf{a}-\mathbf{1 3} \mathbf{a})$ mimicking derivatives $\mathbf{5 a}, \mathbf{2} \mathbf{a}$ and $\mathbf{4 a}$.

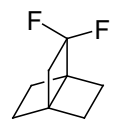

$7 a$

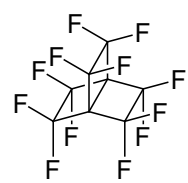

$10 a$<smiles>NC(=O)C1CC2CCC1C2</smiles>

$12 a$

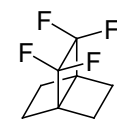

$8 a$

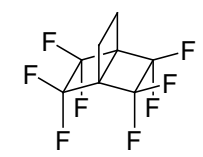

9a

\section{COMPUTATIONAL DETAILS}

Bond-stretch reaction coordinate was defined as stretching of the central carbon-carbon bond (C1-C4) between ca 1.5 to $2.6 \AA$. Calculations were carried using $\operatorname{CASSCF}(2,2) /$ cc-pVDZ method. Active space comprised of two orbitals ( $\sigma_{\mathrm{C} 1-\mathrm{C} 4}$ and $\sigma_{\mathrm{C} 1-\mathrm{C} 4} *$ located on the central carbon-carbon bond, Figure 1a) and two electrons. All geometrical parameters except the central $\mathrm{C} 1-\mathrm{C} 4$ bond were fully optimized in all structures along the reaction path. Minima and maxima on the scans were re-optimized with no restrictions and the nature of stationary points has been determined by vibrational analysis at the same theoretical level used for geometry optimization.

In order to describe Grob-type fragmentation, the diradical structures were re-optimized by $\operatorname{CASSCF}(4,4)$ / cc-pVDZ method. ${ }^{[18]}$ Active space in these calculations composed of four sigma orbitals (bonding and antibonding, located on the central and one of the peripheral carbon- a)
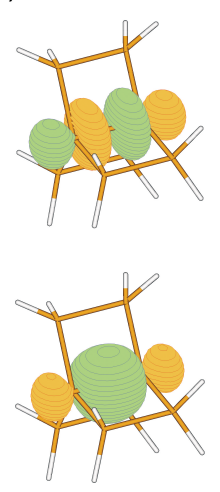

b)

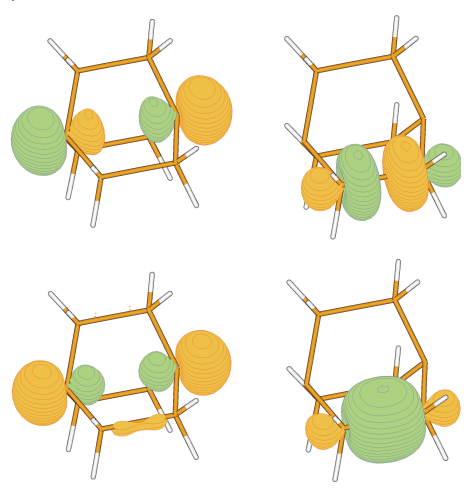

Figure 1. Active orbitals in a) $\operatorname{CASSCF}(2,2)$ and b) $\operatorname{CASSCF}(4,4)$ calculations in closed $1 \mathrm{a}$ and opened $1 \mathrm{~b}$, respectively. 
carbon bonds, see Figure 1b) and four electrons. Reaction coordinate was defined as the peripheral carbon-carbon bond stretching coordinate. As previously applied for bondstretch reaction coordinate, in all points on the scans the structures were optimized with fixed peripheral carboncarbon bond and stationary points were characterized in separated full optimizations. Finally, the energies of all points along scans were refined by CASPT2 ${ }^{[19,20]}$ single point calculations employing cc-pVDZ basis set. The accuracy of applied method has been verified by comparison with high level MR-AQCC calculations available for the parent [2.2.2] propellane. ${ }^{[9]}$ All calculations have been carried out by Molcas programme package ${ }^{[21]}$ and structures and orbitals have been visualized by ZZ-Vega ${ }^{[22]}$ and Molden ${ }^{[23]}$ programs, respectively.

\section{RESULTS AND DISCUSSION}

\section{Validation of the Computational Methods}

As a check of the validity of the results, calculations were first conducted for the parent unsubstituted [2.2.2]propellane 1 and compared with previously published results in Ref. [9] obtained with multireference averaged quadratic coupled cluster (MR-AQCC) method.[24,25] The latter method was proved to provide accurate predictions of the barriers of several bond-stretch isomerisation reactions, ${ }^{[26-29]}$ including the [2.2.2]propellane system in which stretching of the central C1-C4 bond is accompanied by inversion in the occupation of frontier orbitals. However, since our study includes calculations of potential energy paths of a number of rather large molecules to keep the frame of calculations within reasonable limits we decided to use less demanding CASSCF and CASPT2 methods. Further reducing of computational cost was achieved by using minimal active space and basis set of double zeta quality. Comparison of the results obtained in the present study with those from Ref. [9] are summarized in Table 1 and Figure 2. The Cartesian coordinates of all structures optimized by the CASSCF method are provided in Supporting Information.

CASSCF method predicts structure $1 \mathbf{b}$ to be more stable than $1 \mathrm{a}$ by some $5.5 \mathrm{kcal} \mathrm{mol}^{-1}$, whereas the structure 1TSa is higher in energy by 15.1 and $20.6 \mathrm{kcal} \mathrm{mol}^{-1}$ than 1a and $\mathbf{1 b}$, respectively. Comparison of these data with those calculated by MR-AQCC method ${ }^{[9]}$ reveals that relative stability of diradical $\mathbf{1} \mathbf{b}$ with respect to $\mathbf{1 a}$ is more pronounced and that the energy barrier for interconversion between structures $\mathbf{1 a}$ and $\mathbf{1 b}$ is underestimated by $8 \mathrm{kcal} \mathrm{mol}^{-1}$. As previously found at MR-AQCC level, ${ }^{[9,30]}$ the ZPVE correction to the energy barrier at CASSCF level is small. Refinement of the energy of the CASSCF optimized stationary points using CASPT2 method reduces the relative stability of structure $1 \mathrm{~b}$ to $3.2 \mathrm{kcal} \mathrm{mol}^{-1}$ what is in

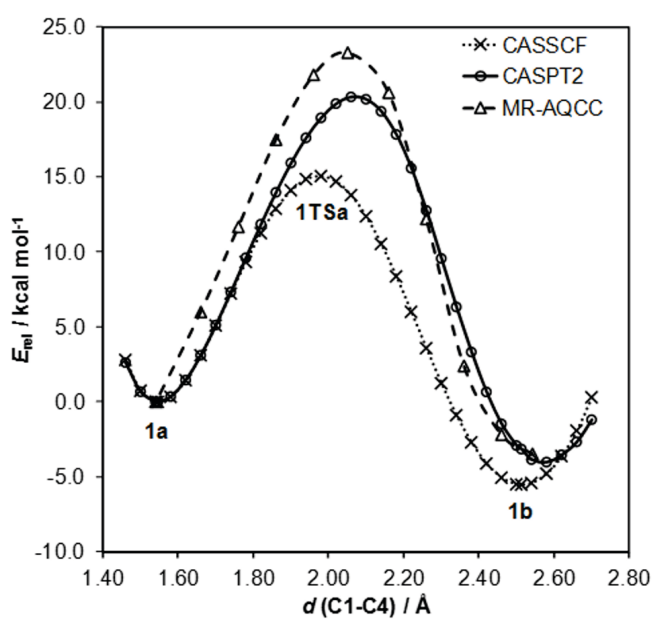

Figure 2. Comparison of the potential energy curves along the C1-C4 stretching reaction coordinate calculated using CASSCF, CASPT2 and MR-AQCC methods. MR-AQCC values are taken from ref [9]. The relative energies are given with respect to the closed structure $1 \mathrm{a}$.

Table 1. Comparison of the relative energies (in $\mathrm{kcal} \mathrm{mol}^{-1}$ ) of the [2.2.2]propellane 1a, saddle points 1TSa and 1TSb and the most stable 1,4-dimethylenecyclohexane isomer relative to the open virtual system $1 \mathbf{b}$ calculated by different theoretical approaches.

\begin{tabular}{|c|c|c|c|c|c|c|}
\hline & Method & $1 a$ & $1 \mathrm{TSa}$ & $1 \mathrm{TSb}$ & $1 c$ & Ref. \\
\hline & MR-AQCC $\left(8,8^{*} ; \mathrm{SA}\right)$ & 3.5 & 26.8 & 1.2 & -42.6 & [9] \\
\hline & MR-AQCC $\left(8,8^{*} ;\right.$ SA)+ZPVE & 3.6 & 26.3 & 0.3 & -44.4 & [9] \\
\hline & CASSCF & 5.5 & 20.6 & 0.4 & -58.4 & This work \\
\hline & CASSCF+ZPVE & 4.6 & 18.8 & -0.4 & -59.8 & This work \\
\hline & CASPT2 & 3.2 & 21.8 & -2.1 & -39.4 & This work \\
\hline & CASPT2(a) & 4.0 & 24.3 & - & - (b) & This work \\
\hline & \multicolumn{6}{|c|}{$\begin{array}{l}\text { The CASPT2 values in minimum and maximum positions on the potential energy surface (Figure 2). The total electronic energy of the reference point with } \\
\text { C1-C4 distance of } 2.55 \AA \text { is }-310.94267 \text { a.u. }\end{array}$} \\
\hline 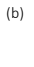 & \multicolumn{6}{|c|}{$\begin{array}{l}\text { When active space is increased from }(2,2) \text { to }(4,4), 1 \mathrm{~b} \text { could not be found as a minimum on the CASPT } 2 \text { potential energy surface, the total electronic energy of } \\
\text { the } 1 \mathrm{c} \text { structure is }-311.00896 \text { a.u. }\end{array}$} \\
\hline
\end{tabular}


good accord with the MR-AQCC prediction. The energy barrier for C1-C4 bond stretching is increased by $3 \mathrm{kcal} \mathrm{mol}^{-1}$ what is again closer to the MR-AQCC result. It should be pointed out that inclusion of the dynamical electronic effects via MR-AQCC method on the geometrical features of the [2.2.2] propellane structures with the C1-C4 distances larger than $1.8 \AA$ are crucial (Figure 2). For example in the minimum 1a both CASSCF and MR-AQCC methods predict very similar C1-C4 distances of 1.546 and $1.542 \AA$, respectively. In the minimum $\mathbf{1 b}$ this is not the case. The C1-C4 distance calculated at the CASSCF level $(2.513 \AA)$ is decreased with respect to the MR-AQCC optimized value ( $2.543 \AA$ ). Furthermore, the maximum of the CASSCF curve is slightly shifted toward shorter C1-C4 distance, i.e. from $2.051 \AA$ at MR-AQCC PE curve to $1.98 \AA$. Therefore, the CASPT2 recalculation of the total electronic energies of the stationary points obtained at the CASSCF potential energy curve could not be expected to provide quite realistic picture of the overall process.

In order to remedy for this drawback and to avoid full optimization of the stationary points at the CASPT2 level of theory, the single point energy for each point on the CASSCF potential energy curve for central bond breaking reaction coordinate were recalculated using CASPT2 method. The so obtained CASPT2 curve turned to be in much better agreement with the reference MR-AQCC curve. Specifically, the barriers for C1-C4 opening in unsubstituted [2.2.2]propellane 1a are 15.1, 20.3 and $23.3 \mathrm{kcal}$ $\mathrm{mol}^{-1}$ at CASSCF, CASPT2 and MR-AQCC level of theory, respectively. The same procedure was applied to calculate CASPT2 potential energy curves in other investigated [2.2.2]propellane derivatives. The points having the lowest and highest energies will be referred as CASPT2 values in further discussion.

It has been shown, both experimentally ${ }^{[11]}$ and theoretically ${ }^{[9]}$ that the open diradical $\mathbf{1} \mathbf{b}$ is elusive intermediate in the process of isomerisation in [2.2.2]propellane as the energy barrier for the breaking of one of the vicinal C-C bonds leading to the 1,4-dimethylene $1 \mathrm{c}$ in its boat conformation is negligible. ${ }^{[9,5]}$ To check whether CASSCF and CASPT2 methods provide the same conclusion as high level MR-AQCC calculations the scan of the- PE surface for rearrangement of $\mathbf{1 b}$ along the $\mathrm{C} 2-\mathrm{C} 3$ stretching reaction coordinate was performed. In these calculations CASSCF method with active space encompassing 4 electrons and 4 orbitals was employed. Subsequently, in all points on the reaction path CASPT2 single point calculations of energies were performed as well (Figure 3 ). Indeed, the energy of the structure $1 \mathrm{TSb}$ is only by $0.4 \mathrm{kcal} \mathrm{mol}^{-1}$ higher than energy of the minimum $\mathbf{1 b}$ and it vanishes by inclusion of ZPVE correction. It should be also noted that on the PE curve calculated by CASPT2 method between 1.5 and $2.0 \AA \AA$ minimum and maximum for $\mathbf{1 b}$ and $\mathbf{1 T S b}$, respectively,

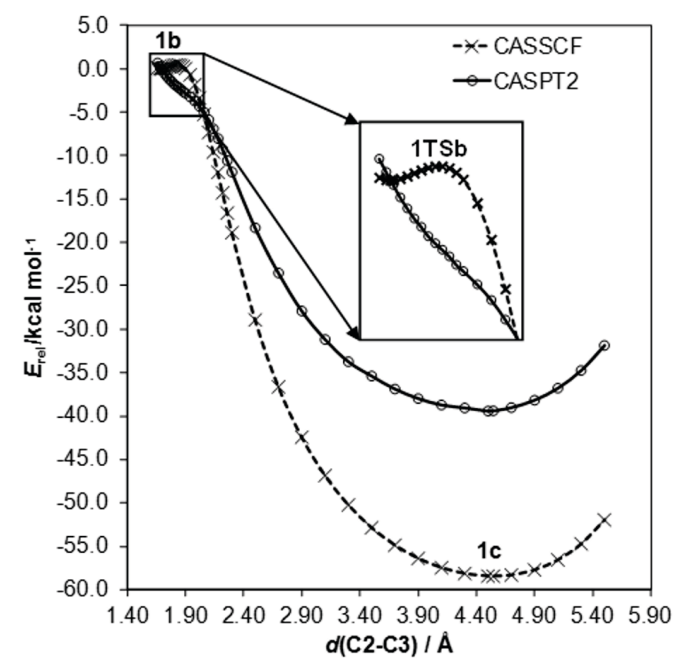

Figure 3. Potential energy curves along the $\mathrm{C} 2-\mathrm{C} 3$ stretching reaction coordinate calculated using CASSCF and CASPT2 methods. The relative energies are given with respect to the structure $1 b$ in the minimum on CASSCF PE surface.

disappear entirely. Instead, a monotonic downhill path is observed. All these findings corroborate conclusion that the diradical $\mathbf{1 b}$ collapses to thermodynamically stable isomer $\mathbf{1 c}$ in a barrierless process, thus providing additional support that methodology utilized here provides a reliable description of [2.2.2]propellane isomerisation.

\section{Isomerisation of Carboxamido Substituted [2.2.2]Propellane}

We next turn to investigation of an amido substitution effect of on the [2.2.2]propellane isomerisation. For the sake of simplicity calculations were carried out on the model structure 12a which mimics synthetically available compound $2 \mathrm{a}$ in which the methyl groups are replaced with hydrogen atoms. Such simplification is expected to have minor effect on the geometrical parameters and relative stability of isomers. Stationary structures for bond-stretch isomerisation in amido substituted [2.2.2] propellane 12a, 12TSa and 12b calculated by CASSCF method are depicted in Figure $4 a$ and the corresponding potential energy profiles at CASSCF and CASPT2 levels are shown in Figure 5a. Since attachment of one substituent makes three peripheral $\mathrm{C}-\mathrm{C}$ bonds unequal preliminary CASSCF scans were done for breaking of all three bonds (C2-C3, C5-C6 and $\mathrm{C7}-\mathrm{C} 8$, respectively). The resulting energy barriers corrected for ZPVEs for the cleavage of the unsubstituted bonds ( $\mathrm{C} 5-\mathrm{C} 6$ and $\mathrm{C} 7-\mathrm{C} 8$ ) were found to be very similar ( 0.63 and $0.65 \mathrm{kcal} \mathrm{mol}^{-1}$, respectively), while for the bond carrying the amido group (C2-C3) a slight increase in barrier of $c a .0 .35 \mathrm{kcal} \mathrm{mol}^{-1}$ was predicted. The CASPT2 energy profiles for stretching $\mathrm{C} 5-\mathrm{C} 6$ bond, which breaks the most 
a) $\operatorname{CASSCF}(2,2)$
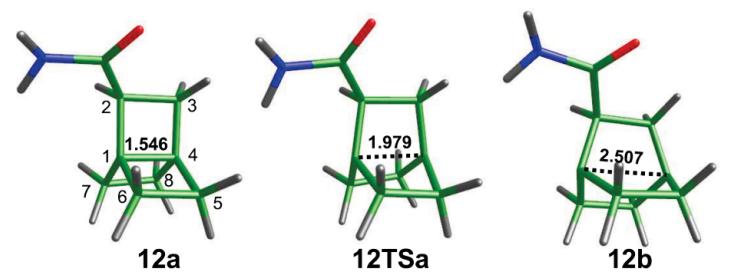

b) $\operatorname{CASSCF}(4,4)$
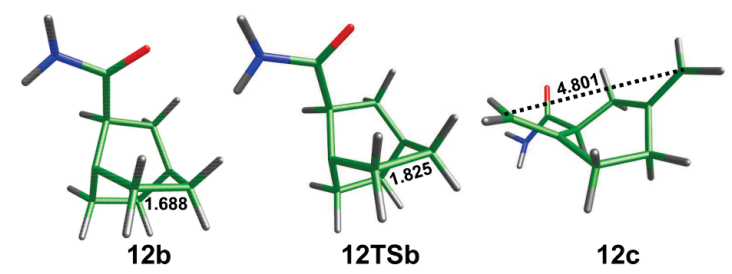

$12 c$

Figure 4. CASSCF optimized structures on PE surface of molecule 12 . Selected bond lengths are given in $\AA$.

easily, is shown in Figure $5 b$ and structures of stationary points $\mathbf{1 2 b}, \mathbf{1 2} \mathrm{TSb}$ and $\mathbf{1 2 c}$ for the reaction are displayed in Figure 4b.

We observe that potential energy profiles for stretching the bond between bridgehead carbons in the parent [2.2.2] propellane and its amido derivative 12a are strikingly similar (Figures 2 and 5a). At the CASSCF level the minimum $12 \mathrm{~b}$ is located at C1-C4 distance of $2.507 \AA$ and it is more stable than 12a with C1-C4 distance of $1.546 \AA$ by some $4.35 \mathrm{kcal} \mathrm{mol}^{-1}$. In analogy to the parent [2.2.2] propellane, relative stability of structure $12 \mathrm{a}$ with respect to $12 \mathrm{~b}$ at CASPT2 level is increased to $-3.14 \mathrm{kcal} \mathrm{mol}^{-1}$. Furthermore, the potential energy well around $\mathbf{1 2 b}$ is shifted toward longer C1-C4 distances with respect to CASSCF and the energy barrier for interconversion of the closed to the diradical form is increased to $20.0 \mathrm{kcal} \mathrm{mol}^{-1}$.

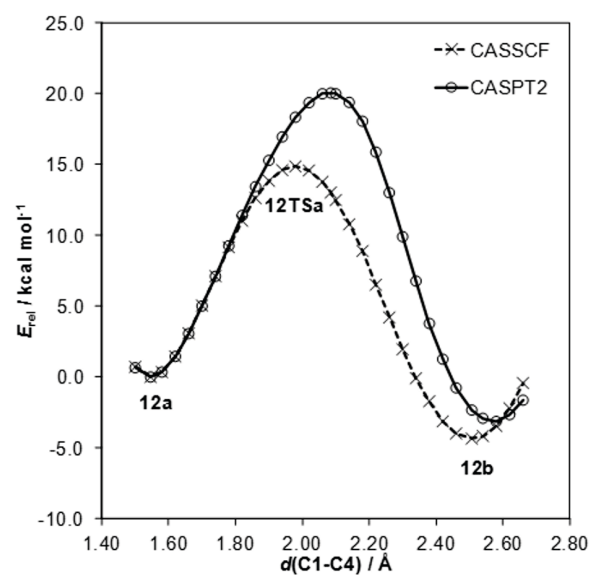

Thus, amido substitution reduces the barrier height only slightly (by $0.3 \mathrm{kcal} \mathrm{mol}^{-1}$ ). Similarity between the profiles for Grob-type opening is also pronounced (Figure 3 and Figure $5 b$ ). Likewise the trend observed for $\mathbf{1 b}$ in Grob-type fragmentation of $\mathbf{1 2 b}$ negligible small barrier on the potential energy profiles for isomerisation of $\mathbf{1 2} \mathbf{b}$ obtained at CASSCF $(4,4)$ level of theory vanished when energies were refined by CASPT2 method. Thus, we can safely conclude that the rearrangement of [2.2.2]propellanes $1 \mathrm{a}$ and $12 \mathrm{a}$ to the corresponding 1,4-dimethylenecyclohexanes 1c and 12c, respectively is controlled by central bond-stretch barrier height. A good agreement of the calculated energy barrier for isomerisation in 12a with experimentally determined value in similar compound $2 \mathrm{a}\left(\sim 22 \mathrm{kcal} \mathrm{mol}^{-1}\right)_{,},[13]$ provides additional support for good performance of the applied computational approach in this case.

Calculations also suggest that introduction of the second carboxamide substituents into 12 a leading to 13 a has only small effect on the reaction profile (see Figure SI1). The energy barrier with respect to $12 \mathrm{a}$ increases by 0.4 and $0.3 \mathrm{kcal} \mathrm{mol}^{-1}$ at CASSCF and CASPT2 levels of theory, respectively. We note in passing that this is in contrast with MNDO results by J. Voss et al. ${ }^{[14]}$ who found an increase of the barrier by $8 \mathrm{kcal} \mathrm{mol}^{-1}$ as compared to $2 \mathrm{a} .{ }^{[14]}$

\section{Effect of Multiple Substitution by Fluorine Atoms on the Ring Opening of [2.2.2] Propellane}

As mentioned above Lemal and coworkers have shown that fluorination alters the character of [2.2.2] propellane fundamentally, transforming it to very resistant toward ring opening. ${ }^{[31]}$ This is in line with well known fact that fluorination has a dramatic effect on stability and reactivities of

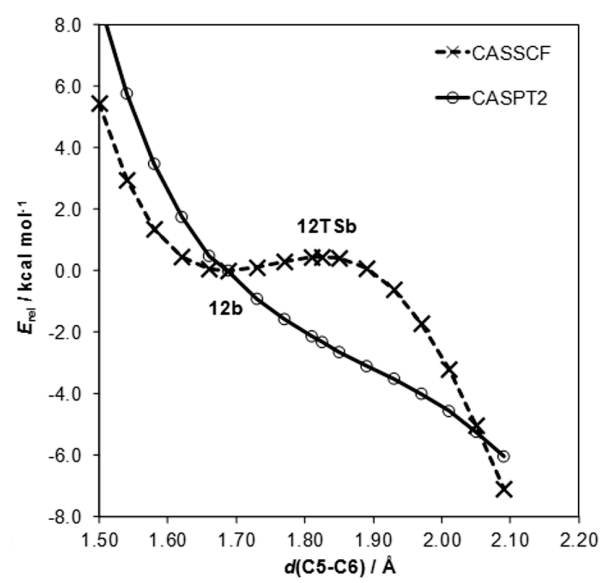

Figure 5. Potential energy curves along the C1-C4 (a) and the C5-C6 (b) stretching reaction coordinate calculated using CASSCF and CASPT2 methods. The relative energies are given with respect to the closed structure $12 \mathrm{a}$ and the opened structure $12 \mathrm{~b}$, respectively, in the minimum on CASSCF PE surface. 
hydrocarbons, ${ }^{[32,33]}$ as well as on radicals with carbon radical centers in syn orientation to eclipsed CF bonds. ${ }^{[34,35]}$ Namely, by replacement of $\mathrm{CH}$ with $\mathrm{CF}$ bonds the net stabilization provided by interaction of carbon radical centers with the filled $\mathrm{CH}$ bonding orbitals is cancelled by hyperconjugative interaction with unfilled CF antibonding orbitals (the $\mathrm{CH}$ bond and the $\mathrm{CF}$ bond are a net hyperconjugative electron donor and net electron acceptor, respectively). ${ }^{[27]}$ The same effects might be expected in case of [2.2.2] propellane structures with strongly stretched C1-C4 central bond and with pronounced diradical character. This surmise was first tested on the compound 7a where one $\mathrm{CH}_{2}$ group was replaced with $\mathrm{CF}_{2}$. Subsequently, $\mathrm{PE}$ curves for bond-stretch reaction coordinate were calculated for multiply fluorinated [2.2.2.]propellane derivatives $\mathbf{8 a}, \mathbf{9 a}$, $6 a$ and 11a as well as for perfluorinated molecule 10a. The results are depicted in Figure 6 .

At the CASSCF level of theory the minimum energy structure for molecule 7a was located at C1-C4 distance of $1.554 \AA$, what is very close to the value found in the parent [2.2.2]propellane 1a. Further replacement of hydrogen atoms with fluorines and functional groups having oxygen atom connected directly to the [2.2.2]propellane skeleton increases the length of the central $\mathrm{C}-\mathrm{C}$ bond, reaching the maximum values of 1.585 and $1.588 \AA$ in $6 a$ and $10 a$, respectively (see Figure 7). Another point worth of noting concerns location of the diradical structure. At the CASSCF level the minimum for structures $\mathbf{7 b} \mathbf{- 9} \mathbf{9}$ and $\mathbf{1 1} \mathbf{b}$, with the inter-bridgehead distances of 2.504, 2.498, 2.435 and $2.417 \AA$, respectively are located. Their energetic ordering relative to the corresponding closed forms is also interesting. The structure of diradical $\mathbf{7 b}$, is found to be ca $7 \mathrm{kcal}$ $\mathrm{mol}^{-1}$ more stable than the $7 \mathrm{a}$, while the structures of the rest of diradicals lie at higher energies than their closed counterparts. For instance, in case of [2.2.2]propellane 8, where net hyperconjugative electron donor stabilization is presumably cancelled by fluorine atoms, the diradical form is by $1.4 \mathrm{kcal} \mathrm{mol}^{-1}$ less stable than $8 \mathrm{a}$. Increasing the number of $\mathrm{H}$ atom substitutions with fluorine causes further increase in energy of the opened form. Specifically, the isomer $\mathbf{9 b}$, is predicted to be by $8.0 \mathrm{kcal} \mathrm{mol}^{-1}$ less stable than $\mathbf{9 a}$ and energy of $\mathbf{1 1} \mathbf{b}$ is only slightly lower than the energy of the transition structure 11TSa. Finally, for molecules $\mathbf{6}$ and $\mathbf{1 0}$ in which all hydrogen atoms are replaced with highly electronegative substituents (fluorine and oxygen) only a plateau for $\mathrm{C} 1-\mathrm{C} 4$ bond stretched structure between 2.2 and $2.4 \AA$ with relative energies around $14 \mathrm{kcal} \mathrm{mol}^{-1}$ are detected.

The CASPT2 results (Figure $6 \mathrm{~b}$ ) offer entirely different picture. Firstly, relative stability of $\mathbf{7 a}$ and $\mathbf{7 b}$ structures is opposite to that found at the CASSCF level - i.e. two fluorine atoms destabilize diradical by $c a .7 \mathrm{kcal} \mathrm{mol}^{-1}$ with respect to the parent [2.2.2]propellane. The minima $\mathbf{8 b}$ and

\section{a) CASSCF}

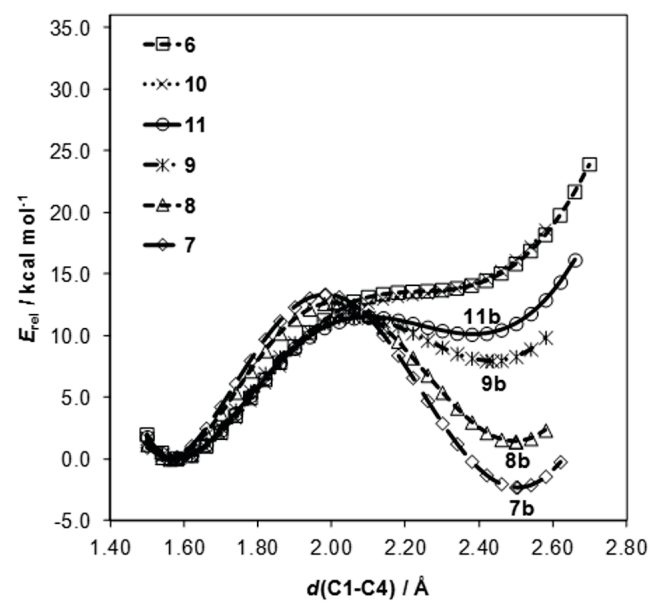

b) CASPT2

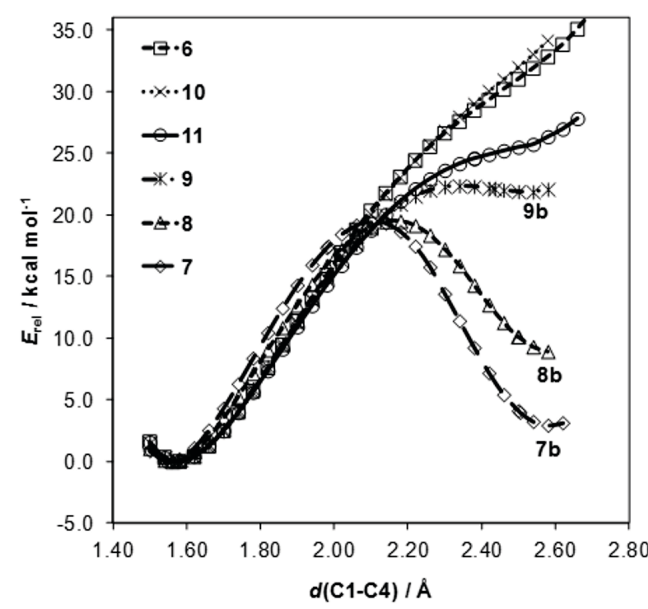

Figure 6. Potential energy curves along the $\mathrm{C} 1-\mathrm{C} 4$ stretching reaction coordinate calculated using a) CASCF and b) CASPT2 methods for multiply fluorinated [2.2.2]propellanes $6 a-11 a$. The relative energies are given with respect to the closed structure.

9b move toward higher energies ( 8.9 and $21.9 \mathrm{kcal} \mathrm{mol}^{-1}$, respectively). The minimum $\mathbf{1 1 b}$ disappeares and the energy of the structure with C1-C4 bond stretched to $2.42 \AA$ is $24.9 \mathrm{kcal} \mathrm{mol}^{-1}$ higher in energy than 11a. Finally, in sharp contrast to CASSCF results, in the rest of considered species $(6,10$ and 11$)$ stretching of the central bond is accompanied by continuous increase in energy, implying that the diradical formation does not take place. In other words in these compounds double well shape of PE curve for bond stretch isomerisation characteristic for parent [2.2.2]propellane is replaced with the single minimum potential energy well. To verify this finding, fully relaxed CASPT2 scan for C1-C4 stretching reaction coordinate was calculated for perfluorinated derivative 10a. Again, the single well shape of PE curve was obtained (Figure SI5). Taking into account better 


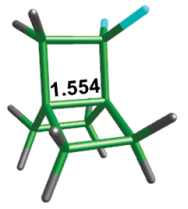

$7 \mathbf{a}$

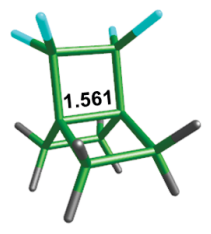

$8 \mathbf{a}$

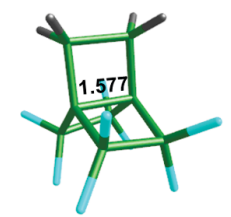

9a

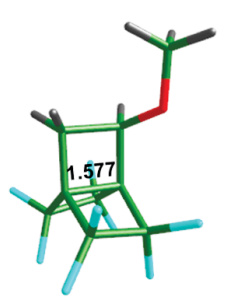

$11 \mathrm{a}$

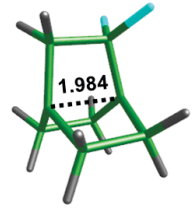

7TSa

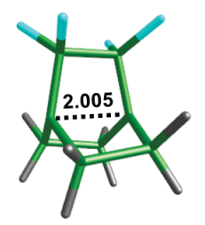

8TSa

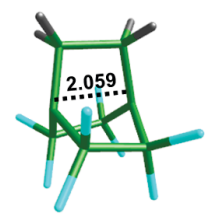

9TSa

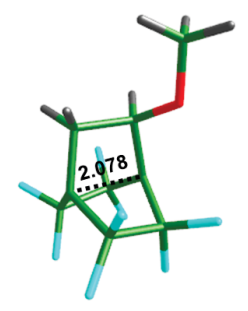

11TSa

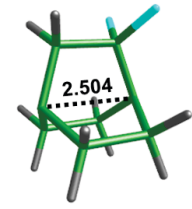

$7 b$

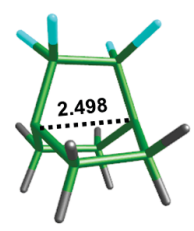

$8 b$

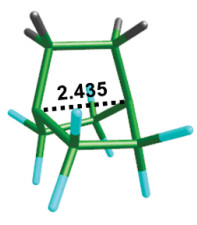

9b

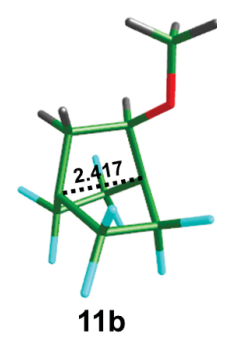

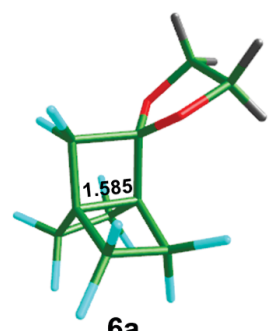

$6 a$

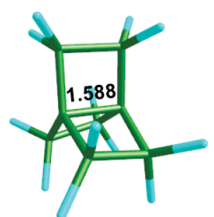

$10 \mathbf{a}$

Figure 7. CASSCF optimized structures on PE surfaces of multiply fluorinated [2.2.2]propellanes. Selected bond lengths are given in $\AA$.

performance of CASPT2 results in describing ring opening of the parent [2.2.2] propellane it is reasonable to conclude that probability of forming diradical in case of poly fluorinated derivatives is highly prohibited. This is in accordance with experimentally observed behaviour of $\mathbf{5 a}$ (here modelled by $9 a$ ) and 6 a.The only exceptions are provided by molecules $\mathbf{7}$ and $\mathbf{8}$ for which local minima corresponding to diradical form were detected. This conclusion, however, could not be proved, since no experimental data on ring opening of these molecules are available at present time.
It is interesting to note that the effect of fluorine substitution on the difference in energy of diradical isomer $\mathbf{b}$ and the closed form a across the series is additive with contributions of 1.7 and $3.2 \mathrm{kcal} \mathrm{mol}^{-1}$ per one substitution (see Figure 8) as calculated by CASSCF and CASPT2 methods, respectively.

For diradicals $\mathbf{7 b}$ and $\mathbf{9 b}$ with two and eight substituents the effect of fluorination on the energy barrier for breaking the vicinal $\mathrm{C}-\mathrm{C}$ bonds is also examined (see Figure 9). The energy of the 7TSb structure for breaking of $\mathrm{CH}_{2}-\mathrm{CF}_{2}$ peripheral bond is found to be $4.63 \mathrm{kcal} \mathrm{mol}^{-1}$ higher in energy than the $\mathbf{7 b}$ minimum at the CASSCF level of theory. The barrier vanishes when CASPT2 method is applied as evidenced by similar change of the shape of PE curve between 1.5 and $2.0 \AA$ as found for the parent diradical $\mathbf{1 b}$ and its amido substituted derivative 12b. The potential energy curves for breaking the $\mathrm{CH}_{2}-\mathrm{CH}_{2}$ bond in $\mathbf{7 b}$ are very similar as for the $\mathrm{CH}_{2}-\mathrm{CF}_{2}$ bond cleavage (see Figure $\mathrm{SI} 3$ ). In the case of more complex derivative $9 \mathbf{b}$, two types of vicinal bonds, i.e. one connecting $\mathrm{CH}_{2}$ groups and the other connecting two $\mathrm{CF}_{2}$ groups, are present. The energy barrier for breaking the $\mathrm{CH}_{2}-\mathrm{CH}_{2}$ peripheral bond on the CASSCF level of theory is almost identical to that in the case of disubstituted derivative $7 \mathrm{~b}\left(3.72 \mathrm{kcal} \mathrm{mol}^{-1}\right)$, whereas on the CASPT2 level of theory it drops to $0.60 \mathrm{kcal} \mathrm{mol}^{-1}$. Potential energy curves along the peripheral $\mathrm{CF}_{2}-\mathrm{CF}_{2}$ bond stretching reaction coordinate (Grob fragmentation) in molecule $\mathbf{9}$ calculated by CASSCF and CASPT2 methods are shown in Figure SI3. Surprisingly, the energy barrier for $\mathrm{CF}_{2}-\mathrm{CF}_{2}$ bond breaking is only slightly higher than for $\mathrm{CH}_{2}-\mathrm{CH}_{2}$ bond (1.6 vs. $0.6 \mathrm{kcal} \mathrm{mol}^{-1}$ at CASPT2 level of theory) in spite of the expected difference in their strength. Still, it can be

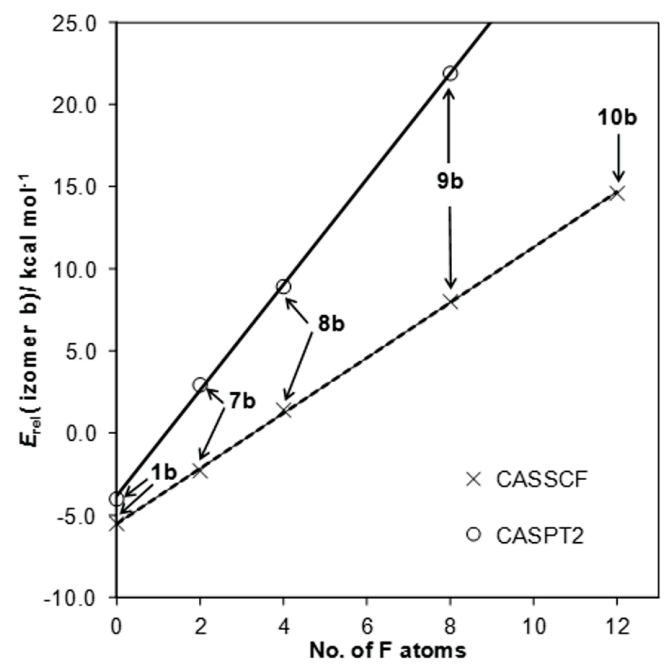

Figure 8. The effect of a fluorine substitution on the relative energy of the open diradical isomer $\mathbf{b}$ with respect to the closed isomer a calculated by CASSCF and CASPT2 methods. 
a)

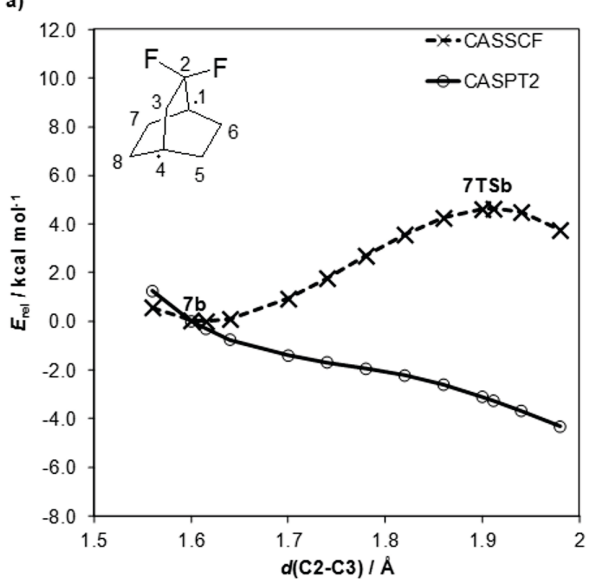

b)

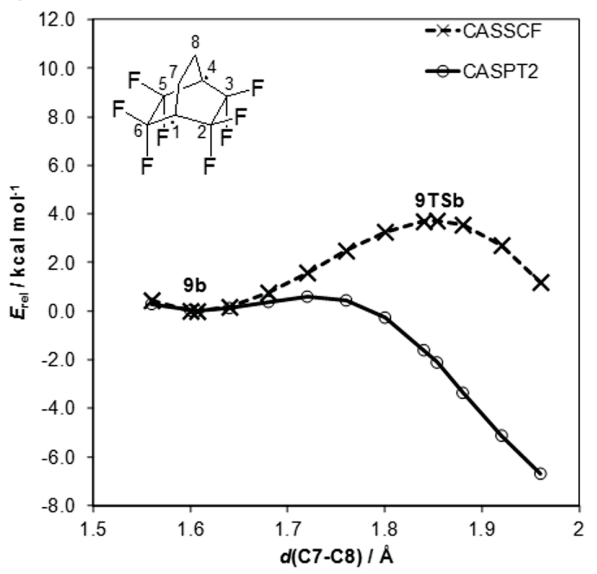

Figure 9. Potential energy curves along the peripheral bond stretching reaction coordinate (Grob fragmentation) for molecules $7 \mathrm{~b}(\mathrm{a})$ and $9 \mathrm{~b}$ (b) calculated by CASSCF and CASPT2 methods. The relative energies are given with respect to the opened structure $7 \mathrm{~b}$ and $9 \mathrm{~b}$, respectively, in the minimum on CASSCF PE surface.

concluded that the increase of substitutions did not strongly influence the cage opening of [2.2.2]propellane diradical to 1,4-dimethylenecyclohexane derivatives. Therefore overall influence of multiple substitution with electron withdrawing groups on the [2.2.2]propellane rearrangement is primarily evidenced in the first step of isomerisation - i.e. cleavage of the central C1-C4 bond.

\section{CONCLUSIONS}

Relative energies of the closed shell minimum and open diradical structure as well as the energy of transition state structure for their interconversion in the parent [2.2.2]propellane and its differently substituted derivatives, were calculated at CASPT2 and CASSCF levels of theory. Comparison of the results with those obtained with MR-AQCC calculations indicated that calculations beyond CASSCF level were needed for reliable description of the process. So obtained results for the parent molecule are in good agreement with values obtained by high level MRAQCC calculations. Barrier height for formation of the diradical structure in amido derivative $12 \mathrm{a}\left(20.0 \mathrm{kcal} \mathrm{mol}^{-1}\right)$ is also in agreement with experimentally determined activation energy for similar $2 \mathrm{a}\left(\sim 22 \mathrm{kcal} \mathrm{mol}^{-1}\right)$, thus confirming the reliability of the chosen method. While replacement of one or two hydrogen atoms with amido or fluorine functional groups did not change PE profiles for isomerisation, CASPT2 calculations show that the effect of multiple substitution with fluorine atoms on the relative energy of the open diradical structure in [2.2.2]propellane is profound. Perfluoro[2.2.2]propellane 10a and multiply fluorinated molecules $6 \mathbf{a}$ and 11a exhibit a single minimum potential energy well, which hampers rearrangement to significantly more stable fluorinated 1,4-dimethylenecyclohexane analogues. The existence of thermally stable compound $\mathbf{6 a}$ is a consequence of increased stability of the closed form with respect to structures with elongated distance between bridgehead carbons. It should be noted that hitherto unsynthesized fully fluorinated derivative 10 a has an almost identical potential energy curve for central bond cleavage. Therefore, its preparation should be feasible if proper synthetic route could be found. Previous attempt to prepare this compound by cycloaddition of tetrafluoroethylene to perfluorobicyclo[2.2.0] hex-1(4)-ene failed presumably due to high electron deficiency of tetrafluoroethylene. ${ }^{[31]}$

Acknowledgement. This work presents continuation of a larger project on bond-stretch isomerism of organic molecules initiated by late Professor Zvonimir Maksić, which was supported by Ministry of Science, Education and Sports of Croatia through the Project 098-0982933-2920. The calculations were performed on the workstations at the Ruđer Bošković Institute and on the Isabella cluster (isabella.srce.hr) at the University of Zagreb Computing Center (SRCE).

Supporting Information: Energy curves along the C1-C4 stretching reaction coordinate for molecule 13 , potential energy curves along the peripheral $\mathrm{CH}_{2}-\mathrm{CH}_{2}$ and $\mathrm{CF}_{2}-\mathrm{CF}_{2}$ bond stretching reaction coordinate (Grob fragmentation) in molecules 7 and 9, respectively, potential energy curves along C1-C4 stretching reaction coordinate in molecule 10 calculated using fully relaxed CASPT2 $\operatorname{scan}, \operatorname{CASSCF}(2,2)$ and $\operatorname{CASSCF}(4,4)$ Cartesian geometries for all stationary points investigated in this work. Supporting information is attached to the electronic version of the article, available at: http://doi.org/10.5562/cca3172. 


\section{REFERENCES}

[1] W.D. Stohrer, R. Hoffmann, J. Am. Chem. Soc. 1972, 94, 779.

[2] J. J. Dannenberg, T. M. Prociv, J. Chem. Soc., Chem. Commun. 1973, 291.

[3] M. D. Newton, J. M. Schulman, J. Am. Chem. Soc. 1972, 94, 4391.

[4] K. B. Wiberg, J. J. Caringi, M. G. Matturro, J. Am. Chem. Soc. 1990, 112, 5854.

[5] E. R. Davidson, Chem. Phys. Lett. 1998, 284, 301.

[6] V. Polo, J. Andres, B. Silvi, J. Comput. Chem. 2007, 28, 857.

[7] M. Filatov, S. Shaik, J. Phys. Chem. A 2000, 104, 6628.

[8] D. Feller, E. R. Davidson, J. Am. Chem. Soc. 1987, 109, 4133.

[9] I. Antol, M. Eckert-Maksić, H. Lischka, Z. B. Maksić, Eur. J. Org. Chem. 2007, 3173.

[10] C. A. Grob, W. Baumann, Helv. Chim. Acta 1955, 38, 594; C. A. Grob, Angew. Chem. 1969, 81, 543.

[11] J. J. Dannenberg, T. M. Prociv, C. Hutt, J. Am. Chem. Soc. 1974, 96, 913.

[12] K. B. Wiberg, W. E. Pratt, M. G. Matturro, J. Org. Chem. 1982, 47, 2720.

[13] P. E. Eaton, G. H. Temme III, J. Am. Chem. Soc. 1973, 95, 7508.

[14] J. Voss, W. Baum, G. Adiwidjaja, U. Behrens, Z. Naturforsch. 2010, 65b, 1498.

[15] Y. Zhang, J. Smith, D. M. Lemal, J. Am. Chem. Soc. 1996, 118, 9454.

[16] Y. He, C. P. Junk, J. J. Cawley, D. M. Lemal, J. Am. Chem. Soc. 2003, 125, 5590.

[17] J. Liebman, W. R. Dolbier Jr, A. Greenberg, J. Phys. Chem. 1986, 90, 394.

[18] B. O. Roos, P. R. Taylor, P. E. M. Sigbahn, Chem. Phys. 1980, 48, 157.

[19] K. Andersson, P.-Å. Malmqvist, B. O. Roos, A. J. Sadlej, K. Wolinski, J. Phys. Chem. 1990, 94, 5483.
[20] K. Andersson, P.-Å. Malmqvist, B. O. Roos, J. Chem. Phys. 1992, 96, 1218.

[21] Molcas 7.4: F. Aquilante, L. De Vico, N. Ferré, G. Ghigo, P.-Å Malmqvist, P. Neogrády, T. B. Pedersen, M. Pitonak, M. Reiher, B. O. Roos, L. Serrano-Andrés, M. Urban, V. Veryazov, R. Lindh, J. Comp. Chem. 2010, 31, 224.

[22] A. Pedretti, L. Villa, G. Vistoli, J. Comput. Aided Mol. Design. 2004, 18, 167; A. Pedretti, L. Villa, G. Vistoli, J. Mol. Graph. 2002, 21, 47; A. Pedretti, L. Villa, G. Vistoli, Theor. Chem. Acc. 2003, 109, 229.

[23] G. Schaftenaar, J. H. Noordik, J. Comput. Aided Mol. Design. 2000, 14, 123.

[24] P. G. Szalay, R. J. Bartlett, J. Chem. Phys. 1995, 103, 3600.

[25] P. G. Szalay, R. J. Bartlett, Chem. Phys. Lett. 1993, 214, 481.

[26] M. Eckert-Maksić, M. Vazdar, M. Barbatti, H. Lischka, Z. B. Maksić, J. Chem. Phys. 2006, 125, 064310.

[27] M. Eckert-Maksić, M. Barbatti, H. Lischka, Z. B. Maksić, M. Vazdar, J. Phys. Chem. A 2009, 113, 8351.

[28] I. Antol, M. Eckert-Maksić, H. Lischka, Z. B. Maksić, ChemPhysChem 2004, 5, 975.

[29] M. Vazdar, M. Eckert-Maksić, Croat. Chem. Acta 2015, 88, 495.

[30] A. R. Gregory, M. N. Paddon-Row, J. Am. Chem. Soc. 1976, 98, 7521.

[31] D. M. Lemal, X. Chen in: The Chemistry of Cyclobutanes, Part 2 (Eds.: Z. Rappoport, J. Liebman) J. Wiley\&Sons, Ltd, 2005, pp. 955-1029.

[32] B. E. Smart in Molecular Structure and Energetics, Vol 3. (Eds.: J. E. Liebman, A. Greenberg) VCH Publishers, Inc.) 1986, pp. 141-191.

[33] D. M. Lemal, J. Org. Chem. 2004, 69, 1.

[34] G. R. Shelton, D. A. Horvat, H. Wei, W.T. Borden, J. Am. Chem. Soc. 2006, 128, 12020.

[35] E. V. Anslyn, D.A. Dougherty, Modern Physical Organic Chemistry; University Science Books; Sausilito, CA, 2006, pp. 120-124. 